\title{
Feasibility and Safety of Uninterrupted Dabigatran Therapy in Patients Undergoing Ablation for Atrial Fibrillation
}

\author{
Tomoyuki Nagao ${ }^{1}$, Yasuya Inden ${ }^{1}$, Masayuki Shimano ${ }^{1}$, Masaya Fujita ${ }^{1}$, \\ Satoshi Yanagisawa ${ }^{1}$, Hiroyuki Kato ${ }^{1}$, Shinji Ishikawa ${ }^{1}$, Aya Miyoshi ${ }^{1}$, \\ Satoshi Okumura ${ }^{1}$, Shiou Ohguchi ${ }^{1}$, Toshihiko Yamamoto ${ }^{1}$, \\ Naoki Yoshida ${ }^{1,2}$, Makoto Hirai ${ }^{3}$ and Toyoaki Murohara ${ }^{1}$
}

\begin{abstract}
Objective Uninterrupted oral warfarin strategy has become the standard protocol to prevent complications during catheter ablation (CA) for the treatment of atrial fibrillation (AF). However, little is known about the safety and efficacy of uninterrupted dabigatran therapy in patients undergoing CA for AF. Therefore, this study investigated the safety and efficacy of uninterrupted dabigatran therapy and compared the findings with those for uninterrupted warfarin therapy.

Methods Bleeding and thromboembolic events during the periprocedural period were evaluated in 363 consecutive patients who underwent CA for AF at Nagoya University Hospital, and received uninterrupted dabigatran $(n=173)$ or uninterrupted warfarin $(n=190)$ for periprocedural anticoagulation.

Results A total of $27(7 \%)$ patients experienced either bleeding or thromboembolic complications. Major bleeding complications occurred in $2(1 \%)$ patients in the dabigatran group (DG) and $2(1 \%)$ patients in the warfarin group (WG). Eight $(5 \%)$ patients in the DG and $9(5 \%)$ patients in the WG experienced groin hematoma, a type of minor bleeding complication. Meanwhile, no patient in the DG and $1(1 \%)$ in the WG developed cerebral ischemic stroke. Overall, there was no significant difference between the groups for any category. The activated partial thromboplastin time (APTT) independently predicted periprocedural complications in the DG.

Conclusion Uninterrupted dabigatran therapy in CA for AF thus may be a safe and effective anticoagulant therapy, and appears to be closely similar to continuous warfarin; however, it is essential to pay close attention to the APTT values when using dabigatran during CA.
\end{abstract}

Key words: dabigatran, atrial fibrillation, catheter ablation

(Intern Med 54: 1167-1173, 2015)

(DOI: 10.2169/internalmedicine.54.3520)

\section{Introduction}

Catheter ablation (CA) has generally been established as the standard therapy for atrial fibrillation (AF) $(1,2)$, and its popularity is gradually increasing (3). However, stroke and thromboembolism can be serious periprocedural complications following left atrial ablation (3). AF ablation utilizing uninterrupted dose-adjusted warfarin has been shown to be safe and it is associated with fewer major bleeding complications $(4,5)$ than an approach using low molecular-weight heparin while bridging with discontinuous warfarin (6). Because in interrupted anticoagulation therapy for AF ablation, heparin is used as an anticoagulant during the periprocedural period, in particular, against an activated coagulation cascade (7), we have to simultaneously be on the lookout for both thromboembolic complications and bleeding complications.

${ }^{1}$ Department of Cardiology, Nagoya University Graduate School of Medicine, Japan, ${ }^{2}$ Division of Cardiovascular Disease, University of Alabama at Birmingham, U.S.A. and ${ }^{3}$ Nagoya University Graduate School of Health Science, Japan

Received for publication June 21, 2014; Accepted for publication September 16, 2014

Correspondence to Dr. Yasuya Inden, inden@med.nagoya-u.ac.jp 
Dabigatran, an oral direct thrombin inhibitor, has been approved as a new oral anticoagulant for the prevention of stroke in patients with nonvalvular $\mathrm{AF}$ as an alternative to warfarin (8). However, whether dabigatran is as safe and efficacious as warfarin for periprocedural anticoagulation therapy in AF ablation remains controversial. Some studies have shown the safety and feasibility of periprocedural dabigatran use during ablation for $\operatorname{AF}(9,10)$, while others found dabigatran to be associated with a higher frequency of periprocedural embolic events than warfarin (11), which is consistent with the published findings of metaanalyses $(12,13)$. In addition, this controversy might result from the various time points at which dabigatran was withheld or restarted (9-11) and while also possibly depending on the interrupted dabigatran strategy for left atrial ablation compared with uninterrupted warfarin therapy (9-11). Three meta-analyses showed that dabigatran use to be equally safe and effective in comparison to warfarin use (14-16). However, the effectiveness of dabigatran use still remains unclear because the protocols for dabigatran use differ from trial to trial (14-16).

We therefore propose a new strategy of uninterrupted dabigatran administration throughout the AF ablation procedure since this strategy has not yet been fully investigated. The aim of this study is to evaluate the safety and efficacy of uninterrupted periprocedural dabigatran compared with uninterrupted warfarin therapy and to identify any possible risk stratification factors for periprocedural dabigatran use.

\section{Materials and Methods}

\section{Study subjects}

We conducted a retrospective chart review of 400 consecutive patients who underwent radiofrequency $\mathrm{CA}$ for $\mathrm{AF}$ at the Nagoya University Hospital between April 2012 and November 2013. All patients provided their written informed consent. At least 4 weeks prior to CA, 190 patients received dabigatran at a dose of 110 or $150 \mathrm{mg}$ twice daily, and 210 patients received dose-adjusted warfarin. The exclusion criteria were as follows: age $>75$ years; creatinine clearance $<30 \mathrm{~mL} / \mathrm{min}$ (17). The dose of dabigatran was determined according to the renal function or age. A low dose of dabigatran (110 mg twice daily) was administered to patients who had the following conditions: mild renal dysfunction [creatinine clearance (Ccr), 30-50 $\mathrm{mL} / \mathrm{min}$ ], advanced age (70-75 years), a past history of upper gastrointestinal ulcer, or the coadministration of glycoprotein inhibitors (amiodarone or verapamil). On the other hand, the warfarin dose was adjusted to maintain a target international normalized ratio (INR) of 1.6-2.6 for older patients (70-75 years) and 2.0-3.0 for younger recipients (younger than 70 years). Dabigatran was administrated twice a day at $7 \mathrm{AM}$ and 7 PM Warfarin and dabigatran were continued throughout the periprocedural period, respectively. Furthermore, the patients in the dabigatran group (DG) received morning and evening dose on the day of the procedure as usual. Heparin was used only during the CA procedure. All patients continued taking each agent for at least 3 months after the CA procedure.

\section{Ablation procedure}

All patients were admitted to our hospital on the day before undergoing the CA procedure. In both groups, blood samples were obtained from every patient approximately 2 hours after they took the anticoagulant because of the peak plasma concentration of dabigatran (18). In tests of blood coagulation, prothrombin time (PT) and activated partial thromboplastin time (APTT) values were obtained using the STA Neoplastin Plus ${ }^{\circledR}$ (Diagnostica Stago, Asnières, France) and the STA APTT $^{\circledR}$ (Diagnostica Stago) reagent, respectively. Transthoracic and transesophageal echocardiography were performed to confirm the cardiac function and exclude the presence of atrial thrombus just before the CA procedure. The morning and afternoon session was started at 9 AM, 1 PM, respectively. Two 8 Fr. sheaths, one 8.5 Fr. steerable sheath, and one 6 Fr. sheath were inserted in the right femoral vein, with one 8 Fr. sheath in the left subclavian vein and one 4 Fr. sheath in the right femoral artery. Intravenous heparin was administered with an initial dose of 5,000 units immediately after inserting the sheaths, the activated clotting time (ACT) was monitored every 30 minutes after the heparin boluses, and additional heparin was administered as necessary with the goal to achieve an ACT of 300 to 350 seconds. After transseptal puncture was performed under intracardiac echocardiography, three sheaths were placed in the left atrium (LA). A circular ring catheter (Lasso $^{\mathrm{TM}}$, Biosense Webster, Diamond Bar, USA) was placed in the ostium of each pulmonary vein (PV) and pulmonary venography was performed for each PV. Encircling PV isolation was performed with a $3.5 \mathrm{~mm}$ tip open-irrigated ablation catheter (Biosense Webster) and the CARTO3 ${ }^{\mathrm{TM}}$ mapping system (Biosense Webster) by the double-Lasso technique (19). The radiofrequency energy output was titrated to $25-35 \mathrm{~W}$ at a flow rate of $17-30 \mathrm{~mL} / \mathrm{min}$, with a maximum temperature of $42^{\circ} \mathrm{C}$. After the successful isolation of all PVs, isoproterenol (5-20 $\mu \mathrm{g} / \mathrm{min})$ was administered, and burst atrial pacing was performed until the shortest cycle length that allowed 1:1 atrial capture from proximal coronary sinus (CS), distal CS, and high right atrium to induce AF. If AF was induced and sustained, then additional substrate modification, including ablation targeting the complex fractionated atrial electrograms, linear ablation of the LA, and isolation of the superior vena cava were performed (20-22). If AF did not subside after these procedures, then the sinus rhythm was restored by internal cardioversion. Cavotricuspid isthmus ablation was performed in all patients (23). Immediately after completion of the CA procedure, protamine $(30-40 \mathrm{mg}$ ) was administered to reverse the effect of heparin and then all the sheaths were removed from the patient. 
Table 1. Patient Characteristics.

\begin{tabular}{|c|c|c|c|}
\hline & $\begin{array}{l}\text { Dabigatran } \\
\mathrm{n}=173\end{array}$ & $\begin{array}{l}\text { Warfarin } \\
\mathrm{n}=190\end{array}$ & $\mathrm{p}$ value \\
\hline Age (years) & $59 \pm 13$ & $59 \pm 9$ & 0.76 \\
\hline Sex (female) & $39(23)$ & $44(23)$ & 0.76 \\
\hline BW (kg) & $67 \pm 13$ & $66 \pm 13$ & 0.54 \\
\hline $\operatorname{BMI}\left(\mathrm{kg} / \mathrm{m}^{2}\right)$ & $24 \pm 4$ & $24 \pm 3$ & 0.67 \\
\hline Non Paroxysmal AF & $49(28)$ & $68(36)$ & 0.08 \\
\hline Coronary artery disease $(\%)$ & $10(6)$ & $7(4)$ & 0.39 \\
\hline Hypertension & $68(39)$ & $63(33)$ & 0.32 \\
\hline Diabetes mellitus & $12(7)$ & $22(12)$ & 0.11 \\
\hline History of heart failure & $12(7)$ & $19(10)$ & 0.25 \\
\hline Prior stroke/TIA & $7(4)$ & $10(5)$ & 0.54 \\
\hline CHADS2 score & $0.6 \pm 0.9$ & $0.7 \pm 0.9$ & 0.55 \\
\hline 0 & $95(55)$ & $97(51)$ & 0.44 \\
\hline 1 & $52(30)$ & $60(31)$ & 0.81 \\
\hline$>=2$ & $26(15)$ & $33(17)$ & 0.52 \\
\hline CHA2DS2-VASc score & $1.2 \pm 1.2$ & $1.3 \pm 1.4$ & 0.25 \\
\hline HAS-BLED score & $0.8 \pm 0.9$ & $0.9 \pm 0.9$ & 0.61 \\
\hline LA size $(\mathrm{mm})$ & $38 \pm 6$ & $39 \pm 7$ & 0.15 \\
\hline LVEF (\%) & $62 \pm 7$ & $61 \pm 7$ & 0.15 \\
\hline LAA velocity $(\mathrm{cm} / \mathrm{s})$ & $65 \pm 24$ & $62 \pm 25$ & 0.50 \\
\hline $\mathrm{BNP}(\mathrm{pg} / \mathrm{mL})$ & $32(16,73)$ & $41(20,83)$ & 0.11 \\
\hline $\operatorname{Ccr}(\mathrm{mL} / \mathrm{min})$ & $96 \pm 35$ & $90 \pm 34$ & 0.11 \\
\hline PT $(\mathrm{sec})$ & $16 \pm 4$ & $23 \pm 5$ & $<0.001$ \\
\hline INR & $1.3 \pm 0.4$ & $2.2 \pm 0.5$ & $<0.001$ \\
\hline $\operatorname{APTT}(\mathrm{sec})$ & $53 \pm 16$ & $44 \pm 8$ & $<0.001$ \\
\hline Fibrinogen $(\mathrm{mg} / \mathrm{dL})$ & $308 \pm 57$ & $316 \pm 71$ & 0.13 \\
\hline D-dimer $(\mu \mathrm{g} / \mathrm{mL})$ & $0.6 \pm 0.2$ & $0.6 \pm 0.4$ & 0.12 \\
\hline \multicolumn{4}{|l|}{ Medication } \\
\hline Aspirin & $14(8)$ & $17(9)$ & 0.69 \\
\hline Clopidogrel & $2(1)$ & $0(0)$ & 0.15 \\
\hline $\mathrm{PPI} / \mathrm{H}_{2} \mathrm{RA}$ & $46(27)$ & $41(22)$ & 0.36 \\
\hline NSAID & $3(2)$ & $2(1)$ & 0.61 \\
\hline AAD & $31(18)$ & $43(23)$ & 0.20 \\
\hline
\end{tabular}

Values are the mean \pm standard deviations (SD) or $\mathrm{n}(\%)$, Abbreviations: BW: body weight, BMI: body mass index, AF: atrial fibrillation, TIA: transient ischemic attack, LA: left atrium, LVEF: left ventricular ejection fraction, LAA: left atrial appendage, BNP: brain natriuretic peptide, Ccr: creatinine clearance, PT: prothrombin time, INR: international normalized ratio, APTT: activated partial thromboplastin time, PPI: proton pump inhibitor, $\mathrm{H} 2 \mathrm{RA}$ : histamine $\mathrm{H}_{2}$-receptor antagonist, NSAID: non-steroidal anti-inflammatory drugs, AAD: antiarrhythmic drug

\section{Postprocedural management and follow-up}

Hematomas and pericardial effusion with or without tamponade were considered to be bleeding complications. Bleeding requiring blood transfusion and pericardial effusion with drainage were classified as major bleeding complications. Small groin hematomas and pericardial effusion that did not require drainage, which were confirmed after the procedure, were classified as minor bleeding episodes. Cerebrovascular accidents, transient ischemic attacks, and systemic emboli were classified as thromboembolic complications after intracranial hemorrhage was ruled out based on the findings of computed tomography. Patients who developed complications received appropriate intervention promptly. Periprocedural complications are defined as adverse events which occur within 30 days before or after the ablation procedure.

\section{Statistical analysis}

All continuous variables are expressed as the mean \pm SD. All categorical variables are reported as the number (per-
Table 2. Procedural Values.

\begin{tabular}{lrrr}
\hline & $\begin{array}{l}\text { Dabigatran } \\
\mathrm{n}=173\end{array}$ & $\begin{array}{l}\text { Warfarin } \\
\mathrm{n}=190\end{array}$ & $\mathrm{p}$ value \\
\hline ACT after heparin bolus (sec) & $278 \pm 68$ & $315 \pm 61$ & $<0.001$ \\
Maximum ACT $(\mathrm{sec})$ & $344 \pm 54$ & $358 \pm 47$ & $<0.05$ \\
Minimum ACT $(\mathrm{sec})$ & $243 \pm 43$ & $270 \pm 37$ & $<0.001$ \\
Mean ACT $(\mathrm{sec})$ & $284 \pm 48$ & $312 \pm 35$ & $<0.001$ \\
procedure time (min) & $153 \pm 46$ & $168 \pm 40$ & 0.79 \\
UFH (U/kg/h) & $62 \pm 29$ & $58 \pm 27$ & $<0.001$ \\
\hline
\end{tabular}

Values are the mean \pm SD, Abbreviations: ACT: activated clotting time, UFH: unfractionated heparin, U: unit

centage) of patients. The unpaired Student's $t$-test was used to analyze variables that exhibit normal distribution to compare continuous variables while Mann-Whitney $U$ test was used for the variables that didn't exhibit it, and categorical variables were compared using either the chi-square test or Fisher exact test. Thereafter, univariate and multivariate logistical regression analyses were performed to evaluate the predictors of complications. Subsequently, the receiveroperating characteristic (ROC) curve was analyzed to determine the optimal cutoff value of APTT. For this cutoff point, the sensitivity and specificity were calculated using standard formulas. A $\mathrm{p}$ value $<0.05$ was considered to be statistically significant. All results were analyzed using the SPSS software program version 18.0 (SPSS, Chicago, USA).

\section{Results}

\section{Baseline characteristics}

A total of 400 patients underwent left atrial CA. Thirtyseven patients $(9 \%)$ were excluded based on the exclusion criteria. There were 173 patients in the DG and 190 patients in the warfarin group (WG). The baseline characteristics of each group are summarized in Table 1. Briefly described, there was no differences were observed with the exception of some factors identified by the anticoagulation test between both groups.

\section{Procedural values}

The procedural values are summarized in Table 2. The ACT after heparin bolus, maximum ACT, minimum ACT, and mean ACT were significantly lower in the DG than in the WG. The procedure time did not differ between the groups. The mean total unfractionated heparin requirement per weight and time was significantly higher in the DG than in the WG.

\section{Complications}

The details of complications during the periprocedural period are shown in Table 3. A total of 27 (7\%) patients had either bleeding complications or thromboembolic complications, with bleeding complications accounting for $26(7 \%)$ of these. Major bleeding complications occurred in 2 (1\%) of 173 patients in the DG and $2(1 \%)$ of 190 patients in the 
Table 3. Complications.

\begin{tabular}{lrrr}
\hline & $\begin{array}{l}\text { Dabigatran } \\
\mathrm{n}=173\end{array}$ & $\begin{array}{l}\text { Warfarin } \\
\mathrm{n}=190\end{array}$ & $\mathrm{p}$ value \\
\hline Total bleeding complications & $12(7)$ & $14(7)$ & 0.89 \\
Major bleeding complications & & & \\
$\quad$ Cardiac tamponade & $2(1)$ & $2(1)$ & 0.93 \\
$\quad$ Vascular complication & $0(0)$ & $0(0)$ & $\mathrm{N} / \mathrm{A}$ \\
Minor bleeding complications & & & \\
$\quad$ Groin hematoma & $8(5)$ & $9(5)$ & 0.96 \\
$\quad$ Pericardial effusion without tamponade & $1(1)$ & $3(2)$ & 0.36 \\
Thromboembolic complications & & & \\
$\quad$ Stroke or TIA & $0(0)$ & $1(1)$ & 0.34 \\
Total complications & $12(7)$ & $15(8)$ & 0.75 \\
\hline
\end{tabular}

Values are $\mathrm{n}(\%)$, Abbreviations: TIA: transient ischemic attack, N/A: not available

WG. Pericardial effusion with tamponade occurred in 2 (1\%) patients in the DG and $2(1 \%)$ patients in the WG. All patients with pericardial tamponade required pericardiocentesis. Pericardiocentesis was successfully performed, and the hemodynamic function was restored in all patients. Minor bleeding complications occurred in $9(6 \%)$ patients in both groups. In the DG, the administration of dabigatran was discontinued for two days in the patients that demonstrated major bleeding complications while it was continued in the patients that showed minor bleeding complications. Thromboembolic complications occurred in $1(1 \%)$ patient in the WG, whereas no thromboembolic complications were observed in the DG. Taken together, there were no significant differences were found between both groups in any of these categories.

\section{Predictor of complications}

We subsequently performed a univariate logistic regression analysis to examine any correlations between the conventional risk factors and complications. The HAS-BLED score was the only predictor of complications in the WG, while APTT and mean ACT were associated with complications in the DG (Table 4). Meanwhile, the start time of session was not predictive of bleeding and/or thromboembolic complications in the DG (Table 4). According to a multivariate logistic regression analysis after controlling for predictive values in univariate analysis, APTT independently predicted the occurrence of periprocedural complications in the DG (Table 5). The distribution of the APTT value was shown in the patients with or without complications (Fig. 1). APTT value was higher than in the patients with complications than in those without complications. The ROC curve was used to estimate the APTT values which predict complications as shown in Fig. 2. The area under the curve (AUC) was 0.86 (95\% CI 0.78-0.94, p<0.001). In addition, Fig. 2 showed that a cutoff value of 65 seconds was able to predict the occurrence of complications with a sensitivity and specificity of $66.7 \%$ and $87.1 \%$, respectively. Meanwhile, the ROC curve analysis of the mean ACT values demonstrated that a cutoff value of 298 seconds (AUC $=0.74$; 95\%CI 0.59 $0.90)$ was able to predict complications with a $75.7 \%$ of sensitivity and a $71.7 \%$ of specificity.

\section{Discussion}

\section{Main findings}

The present study demonstrated no differences in the periprocedural complications between uninterrupted dabigatran use and uninterrupted warfarin use, which thus shows the safety and efficacy of uninterrupted dabigatran in patients undergoing ablation for AF. In addition, APTT independently predicts the occurrence of periprocedural complications in dabigatran therapy.

\section{Periprocedural complications in uninterrupted dabigatran strategy}

The uninterrupted warfarin strategy is currently recommended (4-6) because the CA procedure stimulates thrombogenicity and increases the risk of thromboembolic complications (7). Therefore, adequate anticoagulation therapy is required continuously, not only during the $\mathrm{CA}$ procedure but also before and immediately after the procedure. Given the aforementioned increase in thrombogenicity, discontinuing dabigatran during the periprocedural period may expose CA recipients to temporary suboptimal anticoagulation, especially in cases without heparin-bridging, owing to the short half-life of dabigatran activity (18). This has been reported to result in a higher occurrence of thromboembolic complications than with uninterrupted warfarin treatment (11), and this phenomenon is considered to depend on discontinuing dabigatran during the procedural period. In a meta-analysis comparing most of the patients receiving interrupted dabigatran use with the patients receiving warfarin use, dabigatran use was significantly associated with higher frequency of thromboembolic events (12). In this study, we maintained persistent anticoagulation condition using uninterrupted dabigatran for periprocedural anticoagulation therapy, even if protamine was administered to reverse the effect of heparin immediately after the procedure, with the goal of preventing thromboembolic complications without increasing bleeding complications during the periprocedural period. Recently, Maddox et al. reported a series involving the use of uninterrupted dabigatran throughout the AF ablation procedure as an alternate approach to the use of uninterrupted warfarin (24). Further evaluations are required to determine which method is better: interrupted or uninterrupted dabigatran therapy.

\section{Coping with bleeding during anticoagulant use}

When anticoagulants such as warfarin and dabigatran are used uninterruptedly during the periprocedural period, we have to pay careful attention to bleeding complications. Warfarin has vitamin $\mathrm{K}$ as an antidote, but it takes several hours for its action to take effect. Especially in the case of severe and life-threatening bleeding, fresh frozen plasma, prothrombin complex concentrates and activated recombinant factor VII (rVIIa) are required to cancel the anticoagu- 
Table 4. Univariate Predictors of Complications.

\begin{tabular}{|c|c|c|c|c|c|c|}
\hline & \multicolumn{3}{|l|}{$\begin{array}{l}\text { Dabigatran } \\
\mathrm{n}=173\end{array}$} & \multicolumn{3}{|l|}{$\begin{array}{l}\text { Warfarin } \\
\mathrm{n}=190\end{array}$} \\
\hline & \multicolumn{3}{|l|}{ Complications } & \multicolumn{3}{|l|}{ Complications } \\
\hline & Yes & No & $\mathrm{p}$ & Yes & No & $\mathrm{p}$ \\
\hline No.of patient & 12 & 161 & & 15 & 175 & \\
\hline Age (years) & $63 \pm 12$ & $59 \pm 13$ & 0.27 & $62 \pm 6$ & $59 \pm 9$ & 0.07 \\
\hline Sex: female & $4(33)$ & $35(22)$ & 0.44 & $3(20)$ & $43(25)$ & 0.69 \\
\hline BW (kg) & $62 \pm 16$ & $67 \pm 13$ & 0.13 & $68 \pm 14$ & $66 \pm 14$ & 0.52 \\
\hline $\operatorname{BMI}\left(\mathrm{kg} / \mathrm{m}^{2}\right)$ & $22 \pm 3$ & $24 \pm 4$ & 0.25 & $25 \pm 5$ & $23 \pm 4$ & 0.20 \\
\hline Non Paroxysmal AF & $2(17)$ & $25(16)$ & 0.60 & $6(40)$ & $65(37)$ & 0.63 \\
\hline Coronary artery disease & $0(0)$ & $10(6)$ & 1.0 & $0(0)$ & $6(3)$ & 1.0 \\
\hline Hypertension & $3(25)$ & $65(40)$ & 0.29 & $3(20)$ & $65(37)$ & 0.27 \\
\hline Diabetes mellitus & $0(0)$ & $12(7)$ & 1.0 & $2(13)$ & $20(11)$ & 0.51 \\
\hline History of heart failure & $0(0)$ & $12(7)$ & 1.0 & $0(0)$ & $20(11)$ & 1.0 \\
\hline Prior stroke/TIA & $1(0)$ & $6(4)$ & 0.44 & $1(7)$ & $8(5)$ & 0.44 \\
\hline CHADS2 score & $0.4 \pm 0.7$ & $0.7 \pm 0.9$ & 0.24 & $0.8 \pm 0.9$ & $0.6 \pm 0.8$ & 0.37 \\
\hline 0 & $8(67)$ & $87(54)$ & 0.72 & $7(47)$ & $90(51)$ & 0.44 \\
\hline 1 & $3(25)$ & $49(30)$ & 0.40 & $5(33)$ & $55(31)$ & 0.77 \\
\hline$>=2$ & $1(8)$ & $25(16)$ & 0.54 & $3(20)$ & $30(17)$ & 0.69 \\
\hline CHA2DS2-VASc score & $1.5 \pm 1.4$ & $1.2 \pm 1.2$ & 0.35 & $1.4 \pm 1.4$ & $1.3 \pm 1.3$ & 0.77 \\
\hline HAS-BLED score & $0.8 \pm 0.8$ & $0.8 \pm 0.9$ & 0.96 & $1.3 \pm 0.8$ & $0.7 \pm 0.9$ & $<0.05$ \\
\hline $\mathrm{LA}(\mathrm{mm})$ & $37 \pm 8$ & $39 \pm 7$ & 0.48 & $39 \pm 6$ & $40 \pm 7$ & 0.85 \\
\hline LVEF (\%) & $59 \pm 7$ & $63 \pm 7$ & 0.11 & $61 \pm 6$ & $61 \pm 7$ & 0.96 \\
\hline $\operatorname{Ccr}(\mathrm{mL} / \mathrm{min})$ & $90 \pm 32$ & $97 \pm 35$ & 0.51 & $81 \pm 26$ & $92 \pm 37$ & 0.77 \\
\hline D-dimer $(\mu \mathrm{g} / \mathrm{mL})$ & $0.6 \pm 0.1$ & $0.6 \pm 0.2$ & 0.53 & $0.6 \pm 0.1$ & $0.7 \pm 0.5$ & 0.34 \\
\hline INR & $1.3 \pm 0.2$ & $1.3 \pm 0.4$ & 0.61 & $2.2 \pm 0.5$ & $2.2 \pm 0.7$ & 0.99 \\
\hline APTT(sec) & $71 \pm 18$ & $51 \pm 13$ & $<0.001$ & $47 \pm 9$ & $44 \pm 9$ & 0.23 \\
\hline $\begin{array}{l}\text { ACT after heparin bolus } \\
(\mathrm{sec})\end{array}$ & $315 \pm 80$ & $277 \pm 67$ & 0.50 & $338 \pm 53$ & $313 \pm 60$ & 0.14 \\
\hline Maximum ACT (sec) & $360 \pm 47$ & $345 \pm 45$ & 0.21 & $356 \pm 45$ & $357 \pm 46$ & 0.92 \\
\hline Minimum ACT (sec) & $263 \pm 52$ & $242 \pm 42$ & 0.12 & $286 \pm 46$ & $269 \pm 36$ & 0.08 \\
\hline Mean ACT (sec) & $313 \pm 80$ & $286 \pm 43$ & $<0.05$ & $313 \pm 44$ & $312 \pm 34$ & 0.87 \\
\hline Procedure time (min) & $172 \pm 50$ & $152 \pm 44$ & 0.63 & $138 \pm 40$ & $170 \pm 44$ & 0.47 \\
\hline Morning session & $6(50)$ & $75(47)$ & 0.82 & & & \\
\hline Afternoon session & $6(50)$ & $86(53)$ & 0.82 & & & \\
\hline UFH (U/kg/h) & $50 \pm 33$ & $62 \pm 21$ & 0.12 & $41 \pm 27$ & $48 \pm 22$ & 0.28 \\
\hline \multicolumn{7}{|l|}{ Medication } \\
\hline Aspirin & $0(0)$ & $14(9)$ & 1.0 & $3(20)$ & $13(7)$ & 0.10 \\
\hline Clopidogrel & $0(0)$ & $2(1)$ & 1.0 & 0 & 0 & 1.0 \\
\hline Dabigatran $300 \mathrm{mg}$ & $2(17)$ & $37(23)$ & 0.61 & & & \\
\hline
\end{tabular}

Values are the mean $\pm \mathrm{SD}$ or $\mathrm{n}(\%)$. Abbreviations are summarized in Table 1 and Table 2.

Table 5. Univariate and Multivariate Predictors of Complications in Patients with Dabigatran Use.

\begin{tabular}{lrrrrrrrr}
\hline & \multicolumn{3}{l}{ Univariate Analysis } & & \multicolumn{3}{c}{ Multivariate Analysis } \\
\cline { 2 - 3 } & OR & $95 \% \mathrm{CI}$ & $\mathrm{p}$ value & & OR & $95 \% \mathrm{CI}$ & $\mathrm{p}$ value \\
\hline APTT $(\mathrm{sec})$ & 1.08 & $1.04-1.13$ & $<0.001$ & & 1.09 & $1.04-1.14$ & $<0.001$ \\
Mean ACT $(\mathrm{sec})$ & 1.02 & $1.00-1.04$ & $<0.05$ & & & \\
\hline
\end{tabular}

Values are mean \pm SD or $\mathrm{n}(\%)$ and $95 \%$ confidence interval (CI). OR: odds ratio

Abbreviations are summarized in Table 1 and Table 2.

lant effect. On the other hand, dabigatran does not have any effective antidotes. rVIIa and prothrombin complex concentrates (PCC) might be active for the rapid reversal of dabigatran's effect (25). In addition, a monoclonal antibody has been developed for clinical application (26), although its efficacy remains unclear. Furthermore, we should carefully monitor for the presence of systemic thrombotic emboli because rVIIa and PCC may cause an increased risk of thrombotic complications when the coagulation factors are rapidly replaced during anticoagulation $(27,28)$. There were no pa- tients who required the administration of antidotes, such as coagulation factor complexes, to cancel both drugs during the periprocedural period in this study. Therefore further evaluations are required for the use of rVIIa and PCC.

\section{ACT level during AF ablation}

The concomitant effect of dabigatran and heparin remains unclear. Dabigatran has been shown to prevent ACT from prolongation and it requires a greater amount of heparin administration compared with warfarin in a standard-procedure 


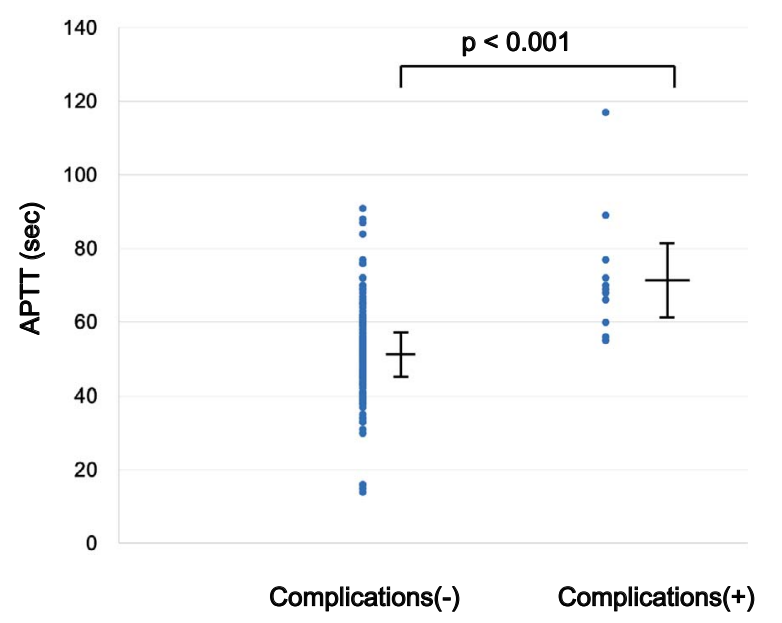

Figure 1. The APTT value and complications. The APTT value is higher in the patients with complications than in those without complications. The error bar indicates the mean \pm SD.

heparin protocol during AF ablation (29). A poor response to heparin with dabigatran is fairly consistent with our results. The ACT level, however, was associated with complications during dabigatran use. In addition, the ROC curve suggested that the mean ACT value of 297 seconds could predict complications with a $75 \%$ of sensitivity and a $71 \%$ of specificity. These results may suggest that we should reconsider heparin administration to maintain a lower target ACT level in an uninterrupted dabigatran strategy than warfarin therapy. We have already understood that openirrigated tip catheters can also allow lower ACT levels (30). However, another report showed that ACT $<300$ seconds predicted the occurrence of silent stroke which was confirmed by performing magnetic resonance imaging after the procedure (31). Therefore, future detailed investigations are required to determine the optimal ACT levels in uninterrupted dabigatran use.

\section{The significance of preoperative APTT values in the DG}

It is crucial for the prevention of bleeding complications to recognize the degree of anticoagulant effect of dabigatran in the preoperative phase because an adequate dose of heparin is used to maintain the ACT levels during the periprocedural period of AF ablation, especially in the patients receiving uninterrupted dabigatran therapy. The ecarin clotting time and thrombin time assay are reported to be useful and highly sensitive as tools for determining dabigatran's anticoagulant effect (32). However, these tests are not generally available in hospital laboratories. Meanwhile, PT or APTT is routinely and easily available at any time. A previous report showed the APTT levels to be correlated with the plasma concentration, although its concentration-response curve was curvilinear (33). This fact suggests that the excessive prolongation of the values causes the occurrence of bleeding events. The present study demonstrated that preoperative high APTT levels (>65 seconds), which correspond to about

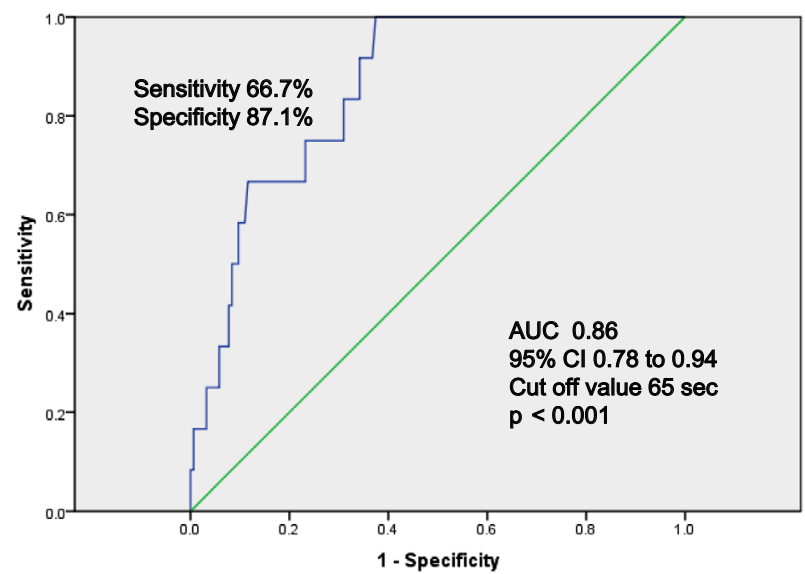

Figure 2. The ROC curve showing the optimal cut off value for APTT to predict complications with a sensitivity of $66.7 \%$ and a specificity of $87.1 \%$. The area under the curve is 0.86 . AUC: area under the curve, CI: confidence interval

double that of the standard value (33 seconds) in this institution, predicted the occurrence of bleeding complications with a high specificity and a relatively low sensitivity. However, further investigation is required with regard to the blood sampling time because dabigatran has an intraindividual variability due to its low bioavailability (34).

\section{Limitations}

There are some limitations associated with this study. First, the present study was a single-center retrospective observational study. Second, older patients with a severe renal function, who had either a high $\mathrm{CHADS}_{2}$ score or HASBLED score, were not sufficiently investigated due to the exclusion criteria (17). In addition, there might have been some selection bias in the two groups due to the research method used in this study. Finally, a larger number of patients would be needed to confirm the efficacy of preventing thromboembolic or bleeding events because the low event rates of complications observed in both groups might have been related to the small sample size.

\section{Conclusion}

The findings of this study suggested that uninterrupted dabigatran use during AF ablation for anticoagulant strategy could maintain the same level of the safety and efficacy as that associated with uninterrupted warfarin use. If we can pay close attention to APTT values, then uninterrupted dabigatran use may become an alternative anticoagulation method with warfarin for AF ablation.

The authors state that they have no Conflict of Interest (COI).

\section{References}

1. Haissaguerre M, Jais P, Shah DC, et al. Spontaneous initiation of atrial fibrillation by ectopic beats originating in the pulmonary 
veins. N Engl J Med 339: 659-666, 1998.

2. Oral H, Knight BP, Tada H, et al. Pulmonary vein isolation for paroxysmal and persistent atrial fibrillation. Circulation 105: 10771081, 2002.

3. Cappato R, Calkins H, Chen SA, et al. Updated worldwide survey on the methods, efficacy, and safety of catheter ablation for human atrial fibrillation. Circ Arrhythm Electrophysiol 3: 32-38, 2010.

4. Wazni OM, Beheiry S, Fahmy T, et al. Atrial fibrillation ablation in patients with therapeutic international normalized ratio: Comparison of strategies of anticoagulation management in the periprocedural period. Circulation 116: 2531-2534, 2007.

5. Hussein AA, Martin DO, Saliba W, et al. Radiofrequency ablation of atrial fibrillation under therapeutic international normalized ratio: A safe and efficacious periprocedural anticoagulation strategy. Heart Rhythm 6: 1425-1429, 2009.

6. Di Biase L, Burkhardt JD, Mohanty P, et al. Periprocedural stroke and management of major bleeding complications in patients undergoing catheter ablation of atrial fibrillation: The impact of periprocedural therapeutic international normalized ratio. Circulation 121: 2550-2556, 2010.

7. Lee DS, Dorian P, Downar E, et al. Thrombogenicity of radiofrequency ablation procedures: What factors influence thrombin generation? Europace 3: 195-200, 2001.

8. Connolly SJ, Ezekowitz MD, Yusuf S, et al. Dabigatran versus warfarin in patients with atrial fibrillation. $\mathrm{N}$ Engl $\mathrm{J}$ Med 361: 1139-1151, 2009

9. Kim JS, She F, Jongnarangsin K, et al. Dabigatran vs warfarin for radiofrequency catheter ablation of atrial fibrillation. Heart Rhythm 10: 483-489, 2013.

10. Kaseno K, Naito S, Nakamura K, et al. Efficacy and safety of periprocedural dabigatran in patients undergoing catheter ablation of atrial fibrillation. Circ J 76: 2337-2342, 2012.

11. Lakkireddy D, Reddy YM, Di Biase L, et al. Feasibility and safety of dabigatran versus warfarin for periprocedural anticoagulation in patients undergoing radiofrequency ablation for atrial fibrillation: Results from a multicenter prospective registry. J Am Coll Cardiol 59: 1168-1174, 2012.

12. Steinberg BA, Hasselblad V, Atwater BD, et al. Dabigatran for periprocedural anticoagulation following radiofrequency ablation for atrial fibrillation: A meta-analysis of observational studies. J Interv Card Electrophysiol 37: 213-221, 2013.

13. Sardar P, Nairooz R, Chatterjee S, Wetterslev J, Ghosh J, Aronow WS. Meta-analysis of risk of stroke or transient ischemic attack with dabigatran for atrial fibrillation ablation. Am J Cardiol 113 1173-1177, 2014.

14. Providencia R, Albenque JP, Combes S, et al. Safety and efficacy of dabigatran versus warfarin in patients undergoing catheter ablation of atrial fibrillation: A systematic review and meta-analysis. Heart 100: 324-335, 2014.

15. Shurrab M, Morillo CA, Schulman S, et al. Safety and efficacy of dabigatran compared with warfarin for patients undergoing radiofrequency catheter ablation of atrial fibrillation: A meta-analysis. Can J Cardiol 29: 1203-1210, 2013.

16. Bin Abdulhak AA, Khan AR, Tleyjeh IM, et al. Safety and efficacy of interrupted dabigatran for peri-procedural anticoagulation in catheter ablation of atrial fibrillation: A systematic review and meta-analysis. Europace 15: 1412-1420, 2013.

17. Eikelboom JW, Wallentin L, Connolly SJ, et al. Risk of bleeding with 2 doses of dabigatran compared with warfarin in older and younger patients with atrial fibrillation: An analysis of the randomized evaluation of long-term anticoagulant therapy (re-ly) trial. Circulation 123: 2363-2372, 2011.
18. Hankey GJ, Eikelboom JW. Dabigatran etexilate: A new oral thrombin inhibitor. Circulation 123: 1436-1450, 2011.

19. Ouyang F, Bansch D, Ernst S, et al. Complete isolation of left atrium surrounding the pulmonary veins: New insights from the double-lasso technique in paroxysmal atrial fibrillation. Circulation 110: 2090-2096, 2004.

20. Nademanee K, McKenzie J, Kosar E, et al. A new approach for catheter ablation of atrial fibrillation: Mapping of the electrophysiologic substrate. J Am Coll Cardiol 43: 2044-2053, 2004.

21. Oral H, Chugh A, Good E, et al. Radiofrequency catheter ablation of chronic atrial fibrillation guided by complex electrograms. Circulation 115: 2606-2612, 2007.

22. Haissaguerre M, Sanders P, Hocini M, et al. Catheter ablation of long-lasting persistent atrial fibrillation: Critical structures for termination. J Cardiovasc Electrophysiol 16: 1125-1137, 2005.

23. Tada H, Oral H, Sticherling C, et al. Double potentials along the ablation line as a guide to radiofrequency ablation of typical atrial flutter. J Am Coll Cardiol 38: 750-755, 2001.

24. Maddox W, Kay GN, Yamada T, et al. Dabigatran versus warfarin therapy for uninterrupted oral anticoagulation during atrial fibrillation ablation. J Cardiovasc Electrophysiol 24: 861-865, 2013.

25. Pernod G, Albaladejo P, Godier A, et al. Management of major bleeding complications and emergency surgery in patients on long-term treatment with direct oral anticoagulants, thrombin or factor-Xa inhibitors: Proposals of the working group on perioperative haemostasis (GIHP) - March 2013. Arch Cardiovasc Dis 106: 382-393, 2013.

26. Ellis CR, Kaiser DW. The clinical efficacy of dabigatran etexilate for preventing stroke in atrial fibrillation patients. Vasc Health Risk Manag 9: 341-352, 2013.

27. Mayer SA, Brun NC, Begtrup K, et al. Efficacy and safety of recombinant activated factor vii for acute intracerebral hemorrhage. N Engl J Med 358: 2127-2137, 2008.

28. Brody DL, Aiyagari V, Shackleford AM, Diringer MN. Use of recombinant factor viia in patients with warfarin-associated intracranial hemorrhage. Neurocrit Care 2: 263-267, 2005.

29. Konduru SV, Cheema AA, Jones P, Li Y, Ramza B, Wimmer AP. Differences in intraprocedural acts with standardized heparin dosing during catheter ablation for atrial fibrillation in patients treated with dabigatran vs. patients on uninterrupted warfarin. J Interv Card Electrophysiol 35: 277-284; discussion 284, 2012.

30. Winkle RA, Mead RH, Engel G, Patrawala RA. Safety of lower activated clotting times during atrial fibrillation ablation using open irrigated tip catheters and a single transseptal puncture. Am J Cardiol 107: 704-708, 2011.

31. Di Biase L, Gaita F, Toso E, et al. Does periprocedural anticoagulation management of atrial fibrillation affect the prevalence of silent thromboembolic lesion detected by diffusion cerebral magnetic resonance imaging in patients undergoing radiofrequency atrial fibrillation ablation with open irrigated catheters? Results from a prospective multicenter study. Heart Rhythm 11: 791-798, 2014.

32. Stangier J, Rathgen K, Stahle H, Gansser D, Roth W. The pharmacokinetics, pharmacodynamics and tolerability of dabigatran etexilate, a new oral direct thrombin inhibitor, in healthy male subjects. Br J Clin Pharmacol 64: 292-303, 2007.

33. van Ryn J, Stangier J, Haertter S, et al. Dabigatran etexilate--a novel, reversible, oral direct thrombin inhibitor: Interpretation of coagulation assays and reversal of anticoagulant activity. Thromb Haemost 103: 1116-1127, 2010.

34. Stangier J, Clemens A. Pharmacology, pharmacokinetics, and pharmacodynamics of dabigatran etexilate, an oral direct thrombin inhibitor. Clin Appl Thromb Hemost 15 (Suppl 1): 9S-16S, 2009.

(C) 2015 The Japanese Society of Internal Medicine http://www.naika.or.jp/imonline/index.html 